\title{
MUTUAL CONTRADICTION OF TWO SELF-CONSISTENT ABSTRACTIONS
}

\author{
KATUZI ONO
}

A vast class of abstractions are proved self-contradictory by Russell-type paradoxes in the sense that the negation of any one of them can be proved tautologically. ${ }^{1)}$ On the other hand, there are a vast class of abstractions, each being self-consistent. A simple criterion for abstractions to be self-consistent (a sufficient condition) can be given. However, even a fairly restricted class of abstractions, each satisfying the criterion to be self-consistent, may contradict to each other. ${ }^{2)}$

In this short note, I would like to notice that we can give a simple criterion for abstractions to be self-consistent (in (2)) but we can also give a simple example pair consisting of self-consistent abstractions satisfying the criterion and contradicting to each other (in (3)). Section (1) serves for preparation.

\section{(1) $T F$-invariance}

Let us assume any set-theoretical system having only one primitive notion MEMBERSHIP $\in$ and standing on the lower classical predicate logic or possibly on a weaker logic such as the intuitionistic predicate logic.

If we evaluate every elementary sentence, naturally a sentence of the form $x \in y$, as TRUE, then we can evaluate every sentence of the system as TRUE or as FALSE definitely even when some free variables may occur in the sentence. I call this truth-value evaluation T-EVALUATION, shortly TEV. Likewise, we obtain another truth-value evaluation by evaluating every elementary sentence as FALSE. This is called $F$-EVALUATION, shortly $F E V$.

Received November 29, 1965.

1) In a joint work with my younger colleague M. OHTA, I have given a sufficient condition for abstractions to be self-contradictory. Known Russell-type paradoxes satisfy the condition. The work will be published in the near future.

2) QUINE and HINTIKKA gave an example of a pair of mutually contradictory propositions, the one being a deformation of abstraction and the other being the natural assumption that there are at least two distinct objects. See QUINE, W. V., 'On Frege's way out', Mind, N. S. 64 (1955), 145-159, and HINTIKKA, K. J. J., 'Vicious circle principle and the paradoxes', J. Symb. Log., 22 (1957), 245-249. 
Any relation $(n$-ary $; n=0,1, \ldots)$ is called $T F$-INVARIANT if and only if it takes a common definite truth-value for the both evaluations, $T E V$ and FEV.

There are simple examples of $T F$-invariant relations. Namely, $x \subseteq y$ defined as usual by $(s)(s \in x \rightarrow s \in y)$ is surely $T F$-invariant, and any relation of the form $\mathfrak{U}(x, y, \ldots, z) \wedge x \mp y$ is also $T F$-invariant. Furthermore, any relation expressible exclusively in terms of $T F$-invariant relations is also $T F$-invariant.

It should be noticed that some relation, not $T F$-invariant itself, can be expressed by a $T F$-invariant relations under some assumptions. For example $\varnothing(x)$ defined by $-(\exists s) s \in x$ is not $T F$-invariant, but it can be expressed by the $T F$-invariant relation $(y) x \subseteq y$ as far as $(\exists x) \emptyset(x)$ is assumed.

\section{(2) A criterion for abstractions to be self-consistent}

Let us call any abstraction

$$
(\exists p)(x)(x \in p \equiv \mathfrak{Q}(x))
$$

PROPER if and only if its kernel $\mathfrak{H}(x)$ is $T F$-invariant.

Any proper abstraction is self-consistent. For, any proper abstraction of the form (A) satisfies $T E V$ if $\mathfrak{A}(x)$ is evaluated as TRUE by TEV and $F E V$, and it satisfies $F E V$ if $\mathfrak{A}(x)$ is evaluated as FALSE by the both evaluations. Anyway, any proper abstraction satisfies one of these evaluations, $T E V$ or $F E V$, so it must be self-consistent.

Now, for any set $\subseteq$ of relations, let us call any abstraction of the form (A) an $\subseteq$-ABSTRACTION if and only if $\mathfrak{U}(x)$ is expressed exclusively in terms of the relations in $\subseteq$.

If $\subseteq$ is a set of exclusively $T F$-invariant relations, any $\subseteq$-abstraction is surely proper, so it is self-consistent.

\section{(3) Mutual contradiction of a fairly restricted class of proper abstractions}

If we restrict $\subseteq$ to a very small set of $T F$-invariant relations, the class of $\subseteq$-abstractions becomes very much limited. ${ }^{3)}$ However, even if we restrict $\subseteq$

3) I have suggested to study theoretical systems starting exclusively from @-abstractions for limited sets $\subseteq$ of relations ('On a restricted abstraction principle', spoken at 
to the unit set of simple $T F$-invariant relation $\subseteq$ defined by

$$
x \in y \equiv(x \notin x \wedge x \in y),
$$

we can still find out a pair of $\Im$-abstractions which contradict to each other.

Namely, the unary relation $x \notin x$ which is no $T F$-invariant relation itself can be expressed in terms of $€$ as $(\exists y) x \Subset y$ by making use of the $\Im$-abstraction

$$
(\exists p)(x)(x \in p \equiv(x \subseteq x \rightarrow x \subseteq x)) .
$$

Accordingly, the $\Subset$-abstraction

$$
(\exists p)(x)(x \in p \equiv(\exists y) x \in y)
$$

contradicts with the preceding $\Im$-abstraction, because the latter $\Im$-abstraction is equivalent to the paradoxical abstraction $(\exists p)(x)(x \in p \equiv x \notin x)$ if the former હ-abstraction is assumed.

Mathematical Institute, Nagoya University

the 1965 Annual Meeting of The Mathematical Society of Japan held at Waseda University on May 21, 1965.). Especially, I have suggested to study $\Xi_{0}$-abstractions for the pair set $\S_{0}$ consisting of two relations $\subseteq$ and $\in$ defined by

$$
x \subseteq y \rightleftharpoons(s)(s \in x \rightarrow s \in y) \quad \text { and } \quad x \in y \equiv x \in y \mp x,
$$

because I could show self-consistency of every $\S_{0}$-abstraction and I could also develop a set theory starting exclusively from $\Xi_{0}$-abstractions.

The result of the present paper has been a byproduct of my unsuccessful struggle to find out a suitable system $S$ of relations that makes every $\mathfrak{S}$-abstraction self-consistent and enables to develop a set theory safely starting exclusively from S.abstractions. The example system $\Im$ given in the present paper may be one of the simplest systems which make each 5 -abstraction self-consistent and also make a certain set of $\Xi$-abstractions mutually contradictory. Recently, the system $\Theta_{0}$ above mentioned has been also proved to belong to the same category by Y. INOUE, one of my younger colleagues. 